\title{
Recomendações para o Diagnóstico da Forma Tardia da Doença de Pompe
}

\author{
Diagnosis Recommendations for Late-onset Pompe Disease
}

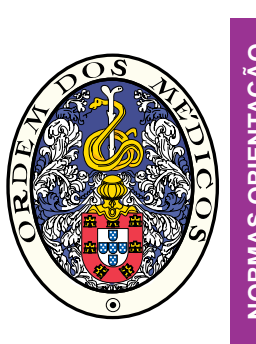

\author{
Luis BRITO-AVÔ', José Delgado ALVES ${ }^{2,3}$, João Matos COSTA ${ }^{4}$, Ana VALVERDE ${ }^{5}$, Lélita SANTOS ${ }^{6}$, Francisco \\ ARAÚJO ${ }^{7}$, Patrício AGUIAR ${ }^{1}$, António MARINHO ${ }^{8}$, Anabela OLIVEIRA ${ }^{1}$, Daniel GOMES ${ }^{1}$ \\ Acta Med Port 2014 Jul-Aug;27(4):525-529
}

\section{RESUMO}

Introdução: A Doença de Pompe é uma miopatia autossómica recessiva progressiva e incapacitante, devida ao défice da enzima lisossómica a-glicosidade-ácida. A sua forma tardia tem uma apresentação heterogénea que mimetiza outras doenças neuromusculares, o que dificulta o diagnóstico.

Objectivo: Desenvolver recomendações baseadas em consenso para o diagnóstico da forma tardia da doença de Pompe.

Material e Métodos: Revisão bibliográfica e análise de um questionário de opinião aplicado a um grupo de especialistas com experiência no diagnóstico de várias miopatias e doenças de sobrecarga lisossomal. Discussão em reunião de consenso.

Recomendações: Doentes com miopatia proximal progressiva, fadiga, cãibras e mialgias devem ser submetidos a uma avaliação complementar com determinação de níveis de creatinina fosfoquinase, electromiograma, espirometria dinâmica e, em casos inconclusivos, biópsia muscular. Nos casos suspeitos ou naqueles em que a biópsia muscular não permita outro diagnóstico deve ser determinada a atividade da enzima lisossómica a-glicosidade-ácida através de teste de gota seca (DBS - dried blood spot). A redução da atividade da enzima lisossómica a-glicosidade-ácida requer a confirmação numa segunda amostra e a sequenciação do gene da enzima lisossómica a-glicosidade-ácida.

Palavras-chave: Idade de Início; Consenso; Doença de Armazenamento de Glicogénio tipo II.

\section{ABSTRACT}

Introduction: Pompe disease is a progressive and debilitating autossomal recessive myopathy due to mutations in lysossomal acida-glucosidase. Its late-onset form has a heterogeneous presentation mimicking other neuromuscular diseases, leading to diagnostic challenge.

Objective: To develop consensus based recommendations for the diagnosis of late-onset Pompe Disease.

Material and Methods: Bibliographic review and analysis of an opinion questionnaire applied to a group of specialists with expertise in the diagnosis of several myopathies and lysossomal storage disorders. Discussed in consensus meeting.

Recommendations: Patients with a progressive limb-girdle weakness, fatigue, cramps and muscle pain should be evaluated with CK levels, electromyography, dynamic spirometry and muscle biopsy in inconclusive cases. Suspected cases and those in which muscle biopsy could not allow other diagnosis should be screened for lysossomal acid-a-glucosidase deficiency with DBS (dried blood spot). The diagnosis should be confirmed by determination of lysossomal acid- $\alpha$-glucosidase activity in a second sample and lysossomal acid-a-glucosidase gene sequencing.

Keywords: Age of Onset; Consensus; Glycogen Storage Disease Type II.

\section{INTRODUÇÃo}

A Doença de Pompe é uma glicogenose /doença de sobrecarga lisossomal causada pela deficiência da enzima lisossomal a-glicosidade-ácida (AGA). Esta deficiência resulta numa acumulação de glicogénio no músculo e outros órgãos com consequente miopatia. ${ }^{1}$

É uma doença autossómica recessiva que resulta da mutação no gene da AGA que se localiza no braço longo do cromossoma 17; já se identificaram mais de 350 mutações. A mutação c. $-32 T>G$ (IVS1-13T > G)é a mais prevalente e ocorre em cerca de $50 \%$ dos doentes Pompe de início tardio nos caucasianos. ${ }^{2}$
O gene da AGA codifica a enzima AGA, responsável pela degradação lisossomal do glicogénio. A mutação do gene condiciona diminuição da atividade da referida enzima, com consequente acúmulo intralisossomal de glicogénio. Esta sobrecarga lisossomal resulta em duas vias patogénicas distintas: rutura da membrana lisossomal (com extravasamento citoplasmático das enzimas hidrolíticas) e alteração do processo de autofagia. ${ }^{3}$

Conhecem-se dois fenótipos distintos que se correlacionam com a atividade da enzima AGA. ${ }^{4} \mathrm{~A}$ forma infantil manifesta-se antes de um ano de idade e caracteriza-se

\footnotetext{
1. Serviço de Medicina 1. Hospital de Santa Maria. Centro Hospitalar Lisboa Norte. Lisboa. Portugal.

2. Serviço de Medicina 4. Hospital Fernando da Fonseca. Amadora. Portugal.

3. Núcleo de Estudos das Doenças Raras. Centro de Estudos de Doenças Crónicas (CEDOC). Faculdade de Ciências Médicas de Lisboa. Lisboa. Portugal.

4. Serviço de Medicina 3. Hospital Distrital de Santarém. Santarém. Portugal.

5. Serviço de Neurologia. Hospital Fernando da Fonseca. Amadora. Portugal.

6. Serviço de Medicina Interna. Centro Hospitalar e Universitário de Coimbra. Coimbra. Portugal.

7. Serviço de Medicina Interna. Hospital Beatriz Ângelo. Loures. Portugal.

8. Unidade de Imunologia Clínica. Centro Hospitalar do Porto. Porto. Portugal.

Recebido: 19 de Fevereiro de 2013 - Aceite: 10 de Julho de 2014 | Copyright $\odot$ Ordem dos Médicos 2014
} 
por uma atividade de $\mathrm{AGA}<1 \%$ do normal, conduzindo à morte por insuficiência cardiorrespiratória geralmente antes do ano de vida. ${ }^{4} \mathrm{~A}$ forma de início tardio manifesta-se após um ano de vida e a atividade da enzima pode variar (1-40\%); a apresentação clínica tende a ser mais heterogénea e a idade de início variável, embora seja uma doença progressiva, que se não tratada poderá conduzir à morte por insuficiência respiratória. ${ }^{5}$

A prevalência na população em geral não é conhecida e varia consoante as formas clínicas de apresentação e os grupos étnicos. A incidência da forma infantil clássica varia desde $1 / 138000$ na população holandesa a 1/33 333 na população de Taiwan. ${ }^{6} \mathrm{~A}$ forma tardia tem uma incidência estimada de $1 / 57000{ }^{6}$ Não existem dados de prevalência na população portuguesa. O único estudo sobre a prevalência de doenças lisossomais na população portuguesa foi realizado entre os anos 1982 e 2001 mas apenas para uma área populacional no Norte, e não para toda a extensão do país, pelo que os dados poderão não ser extrapolados. Considerando os dados existentes a nível internacional, seria expectável uma incidência superior à encontrada, o qual resulta da existência de casos não diagnosticados. Registamos, no entanto, a existência atualmente em Portugal de 27 doentes portadores da doença sob terapêutica enzimática de substituição. ${ }^{7,8}$

A forma tardia da doença de Pompe deverá ser considerada nos adultos que apresentam uma miopatia proximal progressiva, que pode estar associada a insuficiência respiratória de gravidade variável e em qualquer fase da doença, sendo frequentemente uma patologia sub-diagnosticada ou de diagnóstico tardio. ${ }^{9}$ Com este artigo pretende-se alertar para a possibilidade da Doença de Pompe poder ser um dos diagnósticos em casos de miopatias não esclarecidas.

\section{OBJECTIVOS}

O objectivo deste artigo é propor recomendações sob a forma de algoritmo para a identificação precoce de casos da forma tardia da Doença de Pompe na população adulta.

\section{MATERIAL E MÉTODOS}

Foi reunido um painel de especialistas com experiência no diagnóstico e tratamento de várias formas de doenças metabólicas hereditárias e miopatias. Estes realizaram uma revisão da literatura através da pesquisa em bases de dados (PubMed/ Medline/ Cochrane, EMBASE) pelos termos 'Late onset Pompe Disease', 'Adult Pompe Disease', 'Late onset Glycogenosis type Il'. Não foi especificada data limite. Os artigos mais recentes foram selecionados como fonte de evidência. Os especialistas responderam ainda a um questionário anónimo sobre a abordagem diagnóstica do doente com suspeita de miopatia. Os resultados foram posteriormente discutidos em reunião de consenso, onde se concluíram as presentes recomendações.

\section{REVISÃO BIBLIOGRÁFICA - DIAGNÓSTICO Manifestações e Avaliação Clínica}

A forma de início tardio da Doença de Pompe é hete- rogénea, tornando o diagnóstico precoce difícil. O curso clínico é, no entanto, sempre progressivo e a duração da doença não se relaciona com a gravidade da mesma. $O$ tempo médio desde o aparecimento da primeira sintomatologia até ao diagnóstico é variável sendo em média sete anos. $., 10,11$

Os sintomas iniciais podem ser inespecíficos: fadiga generalizada, mialgias e contractura muscular. A forma de apresentação mais comum é o envolvimento proximal dos músculos da cintura pélvica e do tronco com perda da atividade motora grosseira como andar, subir escadas e correr. Ao longo do tempo verifica-se o envolvimento dos músculos da cintura escapular. Também pode surgir, em qualquer fase da doença, fraqueza dos músculos faciais com ptose palpebral uni ou bilateral. ${ }^{5,10-12}$

$\mathrm{Na}$ avaliação de um caso suspeito de Doença de Pompe é importante a avaliação manual da força muscular através de uma escala estandardizada e a realização de testes funcionais básicos (avaliação da marcha e a manobra de Gowers). ${ }^{6,13}$

O diafragma e os músculos axiais paraespinhais são frequentemente envolvidos. Com a progressão da doença pelo menos $1 / 3$ dos doentes acaba por desenvolver insuficiência respiratória. Muitos doentes começam por apresentar alterações ventilatórias durante o período noturno como apneia obstrutiva do sono, hipoventilação, roncopatia, cefaleia e sonolência diurna. Em alguns doentes a paresia do diafragma é a única manifestação clínica. ${ }^{11,14}$

A insuficiência respiratória é a principal causa de morte, estando as anomalias arteriais intracranianas em segundo lugar. ${ }^{11} \mathrm{O}$ envolvimento do músculo liso da parede dos vasos das artérias cerebrais é responsável pelo aumento da incidência de aneurismas cerebrais, sobretudo em território da circulação posterior. ${ }^{15}$

O envolvimento gastrointestinal pode ocorrer sob a forma de dificuldade na mastigação com fadiga dos músculos masseterinos, reflexo faríngeo fraco ou disfagia. ${ }^{4}$

Ao contrário da forma infantil, a cardiomiopatia é rara e pode manifestar-se sob a forma de hipertrofia ventricular direita não relacionada com hipertensão pulmonar e alterações do ritmo (taquicardia sinusal, doença do nódulo sinusal, síndrome de Wolf-Parkinson-White). ${ }^{14}$

Reconhecer precocemente a Doença de Pompe é um desafio uma vez que a semiologia pode ser fruste e de instalação muito progressiva e partilhada com outras doenças neuromusculares. ${ }^{16}$

O diagnóstico diferencial inclui outras miopatias metabólicas (doença de McArdle, miopatias mitocondriais), miopatias inflamatórias, distrofias musculares, miopatias congénitas, doença do neurónio motor ou da placa neuromuscular (Tabela 1). ${ }^{6}$

\section{Exames Complementares}

A elevação da creatinina fosfoquinase (CK) (até 15x o limite superior do normal) tem uma sensibilidade de $95 \%$ mas é inespecífica; o aumento da excreção urinária do tetrassacarídeo-Glc4 pode ser encontrado em qualquer 
doença de armazenamento do glicogénio. ${ }^{4,6,13}$

A espirometria, realizada com o doente sentado e em decúbito dorsal, é útil nomeadamente no estádio pré-sintomático; uma redução da capacidade vital superior a $10 \%$ sugere disfunção do diafragma. ${ }^{6,13}$

O electromiograma (incluindo músculos paravertebrais, sobretudo lombares) revela geralmente um padrão miopático e podem surgir sinais de irritabilidade da membrana, designadamente: atividade insercional aumentada, fibrilhações, ondas positivas ou até descargas miotónicas; os estudos de condução nervosa são normais..$^{6,13}$
A biópsia muscular, que não é obrigatória para o diagnóstico, quando alterada mostra um padrão de miopatia vacuolar; pode, no entanto, ser normal e não específica em $30 \%$ dos casos. ${ }^{6,13}$

O diagnóstico definitivo da Doença de Pompe num doente com quadro clínico consistente com a doença requer a demonstração da redução ou ausência da actividade da AGA ${ }^{6,13}$ Perante a suspeita, deve ser feita a determinação em amostra de sangue seca em cartão próprio (dried blood spot - DBS) para o laboratório de referência. ${ }^{2,6,13}$

Uma actividade da AGA reduzida deve ser confirmada

Tabela 1 - Diagnóstico diferencial da forma tardia da Doença de Pompe (adaptado de Barba-Romero MA et al, 2012)13

$\begin{array}{llll}\text { Grupos de doenças } & \text { Exemplos de doenças } \quad \text { Achados em comum } & \text { Achados diferentes de Pompe }\end{array}$

Distrofias musculares

Distrofia facioescapulo-umeral

Distrofia miotónica-2

Doenças da junção Miastenia gravis, síndrome de neuro-muscular Eaton-Lambert

Doenças do neurónio motor

Atrofia muscular espinhal tipo 3 e 4; Esclerose lateral amiotrófica.

Glicogenoses tipo V e VII

Miopatias congénitas $e$ metabólicas
Doença de Danon (glicogenose Ilb)
Hereditariedade ligada ao X (DMD e DMB); cardiomiopatia.
Fraqueza e atrofia muscular, elevação de CK

Fraqueza muscular proximal subaguda, elevação de CK

Fraqueza intolerância ao elevação de CK

muscular
Fraqueza das cinturas escapulares, escápula alada

Fraqueza muscular proximal fadiga, cãibras, EMG com padrão irritativo, elevação de CK

Fadiga, fraqueza muscular generalizada e dispneia
Envolvimento predominante da musculatura facial.

Hereditariedade autossómica dominante; miotonia clínica; cataratas; insulinorresistência.

MG - Sintomas flutuantes ao longo do dia, envolvimento primordial músculos oculares.

EL - sintomas disautonómicos.

Fasciculações, tremor de acção. ELA - Hipereflexia e reflexos cutâneo-plantares em extensão.

Compromisso sistémico (digestivo cardíaco, cutâneo), autoanticorpos e boa resposta à imunossupressão.

Cardiomiopatia hipertrófica, miopatia vacuolar com acumulação de glicogénio, elevação de CK

Fraqueza muscular intolerância ao exercício elevação de CK
Fraqueza muscular proximal

Miopatias mitocondriais 
numa segunda amostra, nomeadamente em linfócitos purificados de sangue periférico ou em cultura de fibroblastos obtida através de biópsia cutânea. ${ }^{6,13} \mathrm{O}$ estudo genético através da sequenciação do gene AGA deve também ser solicitado. ${ }^{17}$

\section{RECOMENDAÇÕES PARA O DIAGNÓSTICO Clínica}

A suspeita clínica da forma tardia da Doença de Pompe deve ser considerada em qualquer doente, independentemente da idade ou sexo, que apresente:

1. Fraqueza muscular proximal progressiva (mais marcada

\section{SUSPEITA CLÍNICA}

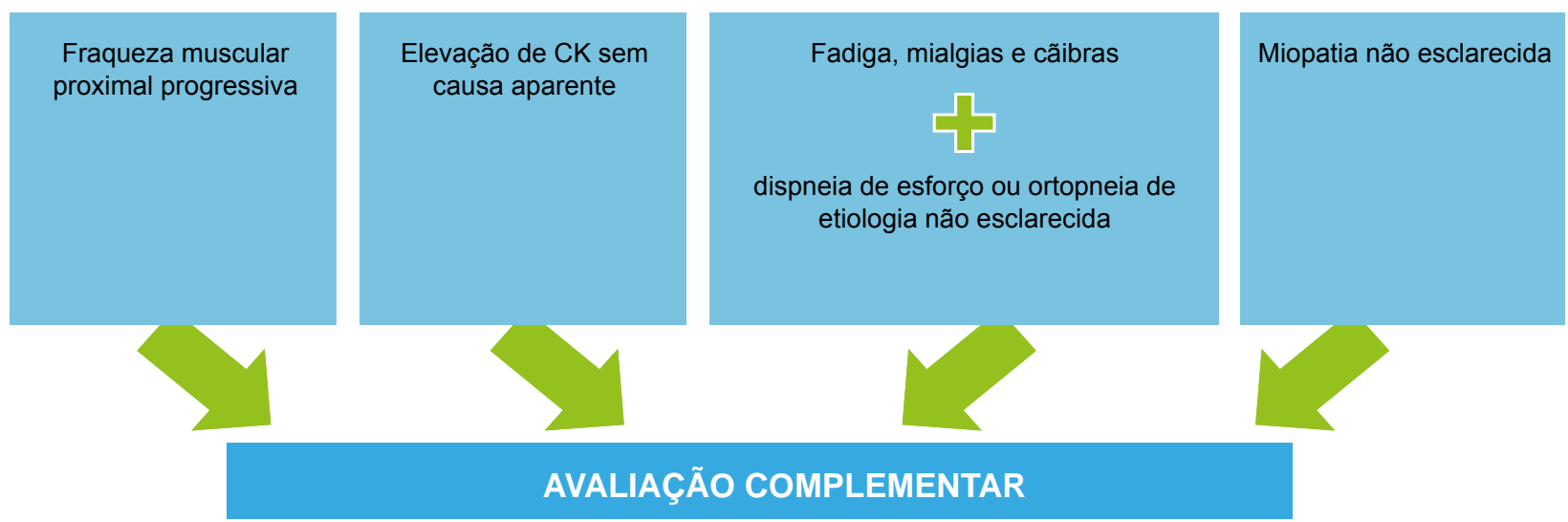

\section{CK (elevado)}

ou

EMG (padrão miopático / irratabilidade + electrofisiologia normal)

ou

Espirometria ( $\downarrow$ CVF $<10 \%$ de sentado para decúbito)

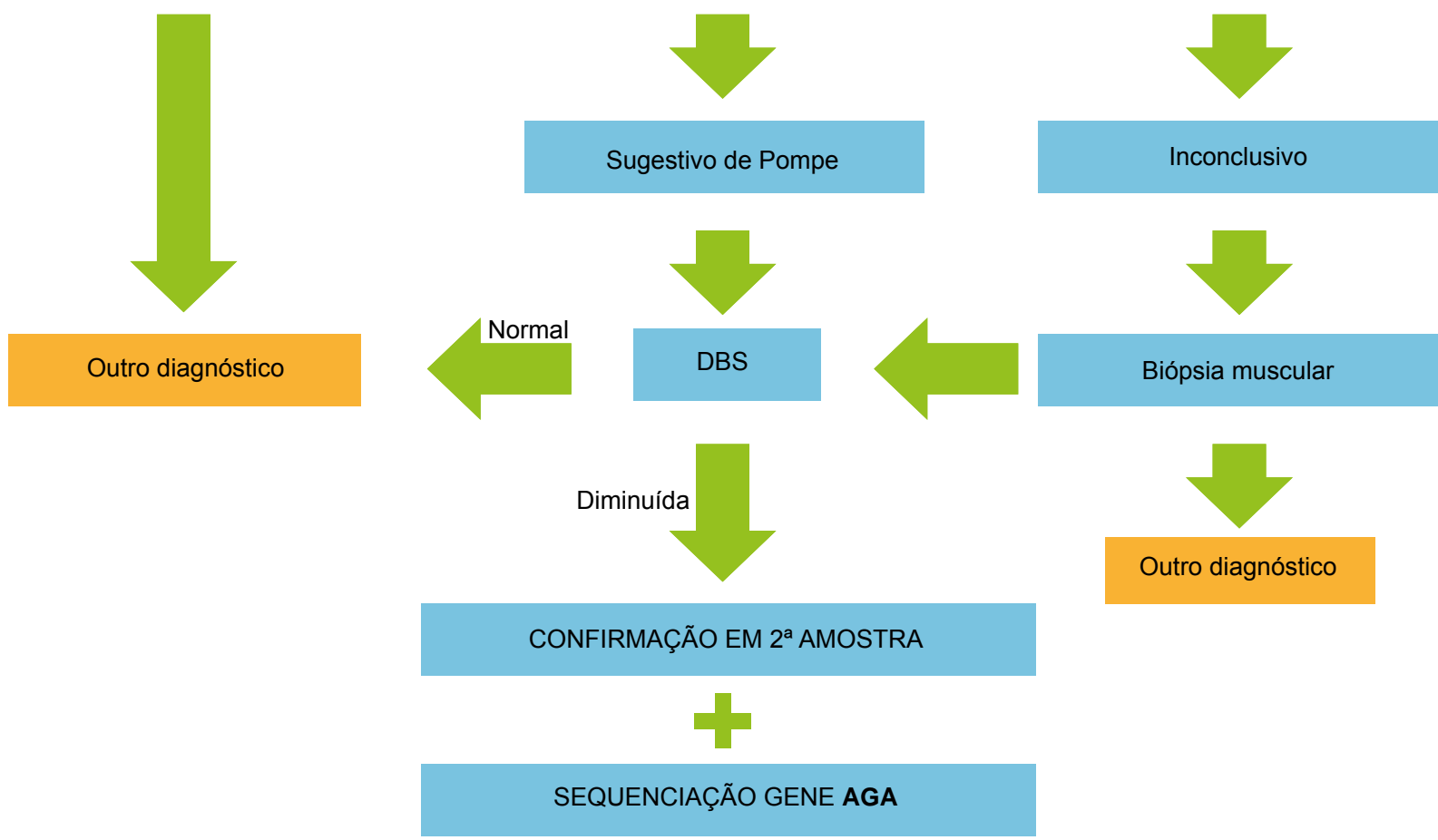

Figura 1 - Algoritmo de diagnóstico para a forma tardia da Doença de Pompe (CK - creatinina fosfoquinase; EMG - electromiograma e estudos de condução nervosa; CVF - Capacidade vital forçada; DBS - dried blood spot; $2^{\mathrm{a}}$ amostra - linfócitos purificados de sangue total ou fibroblastos obtidos por biópsia cutânea; AGA - $\alpha$-glicosidade-ácida). 
na cintura pélvica);

2. Fadiga, mialgias e cãibras;

3. Dispneia de esforço, ortopneia ou apneia noturna.

A ausência de fraqueza muscular proximal não exclui o diagnóstico quando os restantes sintomas estejam presentes.

Os achados no exame objectivo que favorecem o diagnóstico são:

1. Amiotrofia proximal;

2. Hipotonia proximal;

3. Fraqueza de grupos musculares da face e deglutição quando associados a fraqueza muscular proximal.

\section{Estudos Complementares}

Todos os doentes com suspeita clínica devem ser submetidos aos seguintes exames complementares:

1. Níveis séricos da enzimologia muscular (CK, aspartato aminotransferase, aldolase);

2. Estudos de condução nervosa e eletromiograma;

3. Espirometria em posição sentada e decúbito dorsal;

4. Biópsia muscular em casos inconclusivos.

De modo geral, recomendamos que resultados negativos em doentes com quadro clínico sugestivo não devem excluir o diagnóstico, e nestes casos deve prosseguir-se para confirmação diagnóstica (de acordo com capítulo seguinte). ${ }^{18}$

\section{REFERÊNCIAS}

1. Laforêt $P$, Weinstein DA, Smit $G$. The glycogen storage diseases and related disorders. In: Saudubray JM, Berghe G, Walter J, editors. Inborn metabolic diseases. Diagnosis and treatment. Heidelberg: Springer; 2012. p.116-39.

2. Wagner M, Chaouch A, Müller JS, Polvikoski T, Willis TA, Sarkozy A, et al. Presymptomatic late-onset Pompe disease identified by the dried blood spot test. Neuromuscul Disord. 2013;23:89-92.

3. Raben N, Roberts A, Plotz PH. Role of autophagy in the pathogenesis of Pompe disease. Acta Myol. 2007;26:45-8.

4. Kishnani PS, Steiner RD, Bali D, Berger K, Byrne BJ, Case LE, et al. Pompe disease diagnosis and management guideline. Genet Med. 2006;8:267-88.

5. Wokke JH, Escolar DM, Pestronk A, Jaffe KM, Carter GT, van den Berg $\mathrm{LH}$, et al. Clinical features of late-onset Pompe disease: a prospective cohort study. Muscle Nerve. 2008;38:1236-45.

6. American Association of Neuromuscular \& Electrodiagnostic Medicine. Diagnostic criteria for late-onset (childhood and adult) Pompe disease. Muscle Nerve. 2009;40:149-60.

7. Pinto R, Caseiro C, Lemos M, Lopes L, Fontes A, Ribeiro H, et al. Prevalence of lysosomal storage diseases in Portugal. Eur J Hum Genet. 2004;12:87-92

8. Instituto Nacional de Saúde Ricardo Jorge [homepage na Internet]. Doenças lisossomais de sobrecarga (DLS) - Relatório 2011 [consultado 2014 Jan 13]. Disponível em: http://www.insa.pt/.

9. Dubrovsky A, Corderi J, Karasarides T, Taratuto AL. Pompe disease, the must-not-miss diagnosis: a report of 3 patients. Muscle Nerve. 2013;47:594-600.

10. Müller-Felber W, Horvath R, Gempel K, Podskarbi T, Shin Y, Pongratz D,

\section{Confirmação Diagnóstica}

Esta deve ser realizada, em primeira linha, através de DBS (Dried Blood Spot) em cartão enviado para o centro de referência. Um resultado positivo (atividade enzimática reduzida) deve ser confirmado por ambos os métodos:

1. Atividade enzimática numa segunda amostra (linfócitos purificados de sangue periférico ou cultura de fibroblastos em biópsia cutânea);

2. Sequenciação do gene AGA.

\section{CONCLUSÃO}

O diagnóstico da forma tardia da doença de Pompe requer elevada suspeição clínica, podendo em alguns casos ser encontrada na prática clínica. É fundamental estar alerta para a possibilidade do diagnóstico perante um doente com fraqueza muscular proximal progressiva, fadiga generalizada, cãibras e níveis elevados de CK. A confirmação diagnóstica não deve ser atrasada, dado o impacto no prognóstico da terapêutica de substituição enzimática.

\section{CONFLITOS DE INTERESSE}

Os autores declaram não existir quaisquer conflitos de interesse.

\section{FONTES DE FINANCIAMENTO}

Este trabalho foi realizado com o apoio financeiro da Sanofi - Produtos Farmacêuticos, Lda. e não foi condicional à obtenção de nenhum tipo específico de resultados.

et al. Late onset Pompe disease: clinical and neurophysiological spectrum of 38 patients including long-term follow-up in 18 patients. Neuromuscul Disord. 2007;17:698-706.

11. Byrne BJ, Kishnani PS, Case LE, Merlini L, Müller-Felber W, Prasad S, et al. Pompe disease: design, methodology, and early findings from the Pompe Registry. Mol Genet Metab. 2011;103:1-11.

12. Groen WB, Leen WG, Vos AM, Cruysberg JR, van Doorn PA, van Engelen BG. Ptosis as a feature of late onset glycogenosis type II. Neurology. 2006;67:2261-2.

13. Barba-Romero MA, Barrot E, Bautista-Lorite J, Gutierrez-Rivas E, Illa I, Jimenez LM, et al. Clinical guidelines for late-onset Pompe disease. Rev Neurol. 2012;54:497-507.

14. Schüller A, Wenninger S, Strigl-Pill N, Schoser B. Toward deconstructing the phenotype of late-onset Pompe disease. Am J Med Genet C Semin Med Genet. 2012;160C:80-8.

15. Kretzschmar HA, Wagner H, Hübner G, Danek A, Witt TN, Mehraein P. Aneurysms and vacuolar degeneration of cerebral arteries in late-onset acid maltase deficiency. J Neurol Sci. 1990;98:169-83.

16. Kroos MA, Pomponio RJ, Hagemans ML, Keulemans JL, Phipps M, DeRiso $M$, et al. Broad spectrum of Pompe disease in patients with the same c.-32-13T->G haplotype. Neurology. 2007;68:110-5.

17. Pompe Disease Diagnostic Working Group, Winchester B, Bali D, Bodamer OA, Caillaud C, Christensen E, et al. Methods for a prompt and reliable laboratory diagnosis of Pompe disease: report from an international consensus meeting. Mol Genet Metab. 2008;93:275-81.

18. Toscano A, Montagnese F, Musumeci O. Early is better? A new algorithm for early diagnosis in late onset Pompe disease (LOPD). Acta Myol. 2013;32:78-81. 
Luis BRITO-AVÔ, José Delgado ALVES, João Matos COSTA, Ana VALVERDE, Lélita SANTOS, Francisco ARAÚJO, Patrício AGUIAR, António MARINHO, Anabela OLIVEIRA, Daniel GOMES

\section{Recomendações para o Diagnóstico da Forma Tardia da Doença de Pompe Acta Med Port 2014:27:525-529}

Publicado pela Acta Médica Portuguesa, a Revista Científica da Ordem dos Médicos

Av. Almirante Gago Coutinho, 151

1749-084 Lisboa, Portugal.

Tel: +351 218428215

E-mail: submissao@actamedicaportuguesa.com

www.actamedicaportuguesa.com

ISSN:0870-399X | e-ISSN: 1646-0758

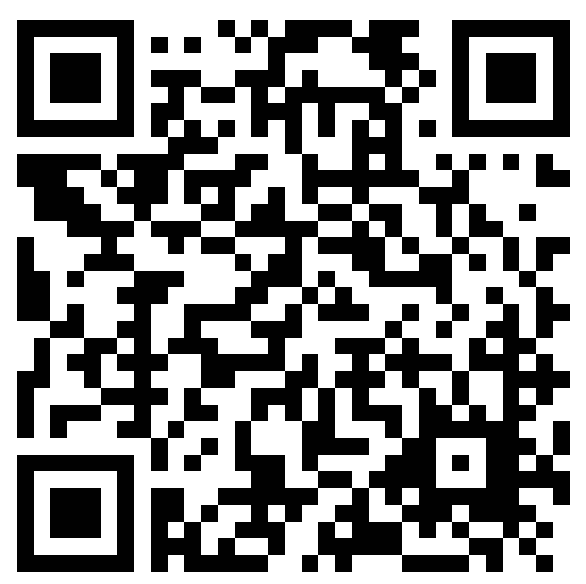

